\title{
Research on the Efficiency of Financial Technology in the Pearl River Delta Region
}

\author{
Hongyi Chen \\ Department of Finance, Southern University of Science and Technology, Shenzhen, China \\ 397004301@qq.com
}

\begin{abstract}
The deep integration of science and financial industry has become an important development strategy for building an innovation-driven country. As the forefront of China's reform and opening up, the Pearl River Delta region shoulders the important task of studying the development and influencing factors of Fin-Tech efficiency. Based on the panel data of nine major cities in the Pearl River Delta region from 2010 to 2018, this paper selects input and output indicators from aspects of manpower and capital, and uses DEA-Malmquist index model to measure the efficiency of Fin-Tech. The results demonstrate that during the period studied, the annual average Fin-Tech efficiency in the Pearl River Delta region presents an alternately fluctuating trend of "updown". Among the inner cities, there are significant differences in the efficiency of Fin-Tech due to different regional development. Moreover, this paper explores the reasons from the aspects of macro economy, policy regulation and local development, and provides corresponding suggestions.
\end{abstract}

Keywords: The Pearl River Delta Region (PRD); Fin-Tech; DEA-Malmquist Index Model.

\section{Introduction}

At the beginning of the 21 st century, China has put forward the development concept of Fin-Tech. According to the research of scholars and experts, the core of Fin-Tech is still finance with the purpose to complete the life cycle of high-tech enterprises, promote the development of scientific research and transform the achievements. The Outline of the National Medium and Long-Term Program for Scientific and Technological Development (2006-2020) issued by the government in 2006 has proposed the development concept of promoting the integration between technology and finance, cultivating strategic emerging industries and leading the transformation of economic development mode. According to this, the countries successively promulgated documents about promoting fin-tech to speed up the implementation of independent innovation strategy in the 12th Five-Year Plan and the 13th Five-Year Plan. In order to accelerate the transformation of technological achievements and benefit people's livelihood, the government has advanced the scientific development from three fields, such as independent innovation, technology competitiveness and international influence. What's more, it is helpful to realize two goals in one hundred and the three-step strategy. In the 19th National Congress of the China Communist Party, it emphasized the development direction of the deep combination between technology and finance. In 2020, the Ministry of Science and Technology and the Postal Savings Bank of China will sign the Strategic Cooperation Agreement on Science and Finance to establish multi-level contact and cooperation to ensure the implementation of national and local science and technology innovation projects, the development of high-tech enterprises and the establishment of technical innovation parks.

The Pearl River Delta Economic Zone (PRD) is an urban cluster which was first proposed in 1994, including Guangzhou, Shenzhen, Foshan, Dongguan, Zhongshan, Zhuhai, Jiangmen, Zhaoqing and Huizhou. The PRD economic zone is located in Guangdong Province. As the leading edge of reform and opening up, its economy has ranked first in China for 29 consecutive years as of 2018, transforming from high-speed growth to high-quality rise. Since its establishment, the PRD has received a lot of policy support and resource tilt from the government of China and Guangdong Province with achieving sustained rapid economic growth, high level of export-oriented economy, and optimization of industrial structure. By 2018, the PRD 's resource input and GDP had already reached more than 50 percent of that of Guangdong. As well as, the overall level of development has surpassed that of the Yangtze River Delta at the top of China's five major urban agglomerations. 
PRD's per capita GDP and urbanization are far ahead of other urban agglomerations. In 2019, the PRD will be closely linked with Hong Kong and Macao officially, undertaking the important development goal of building an international first-class bay area and urban agglomeration.

The technical output and financial development of the PRD have been very outstanding with great strategic significance. Therefore, based on the data of cities in PRD from 2010 to 2018, this paper analyzes its Fin-Tech efficiency, discussing the current possible problems and methods to improve, as well as exploring experience for other places.

\section{Literature Review}

\subsection{Foreign Literature}

Foreign researches concentrate on finance and technology respectively. Sfinga and Niklis (2011) analyzed the impact of the 2008 financial crisis on European commercial banks' efficiency. On account of the DEA model, this paper compared with banks' efficiency before and after the financial crisis with the data from 2006 to 2010, putting forward the suggestion that banks should have the ability and flexibility to fit in with the rapid change. Branda and Kopa (2012) conducted various methods, including DEA model to investigate the factors of world stock indexes. Among them, multiple risk quantitative indicators were used as input indexes and average total return as output index. It came to a conclusion that VaR was the largest influence factor. On the other hand, Seol, Lee et al. (2008) discussed the effect of information technology on the public service efficiency of the South Korean government and optimized the DT model based on DEA model from three aspects: the internal relationship of multiple information technology variables; priority between variables; the possible influence of information technology on the new department. Their research provided recommendations to the government public affairs department, as well as improved information technology efficiency.

\subsection{Domestic Literature}

Firstly, the DEA model is applied to analyze and evaluate the industry efficiency. When it came to assess diversified enterprise policy formulation and resource allocation, Jiang (2020) modified the traditional DEA model in order to avoid the choice and separation of input indexes and made direct weight assignment for it directly, so that it could be closely associated with the industry overall situation and achieve more accurate measurement of different production efficiency between government departments and more efficient allocation of resources. Ren (2020) explored the future development of new energy vehicle companies based on the economic development and environmental protection issues, which have been focused on in recent years. By selecting more than 170 listied companies, the author calculated the situation through DEA-Malmquist and Tobit models with a conclusion that the industry has been on the rise in recent years.

Secondly, previous studies concentrate on the development and effect of Fin-Tech in China. Liu (2020) aimed at small and medium-sized enterprises' financing current difficulties in traditional financial services, and applied the cash flow-cash sensitivity model from three perspectives, namely, the development of Fin-Tech, the perfection of legal system and local economic condition. The author found that financing constraint can be effectively alleviated in these three aspects. Making use of commercial banks' Fin-Tech index from 2011 to 2018, Wang (2020) adopted Long Tail Theory, Destructive Innovation Theory and Technological Innovation Theory to illustrate the of Fin-Tech's mechanism for commercial banks. At the same time, the horizontal and vertical comparison were carried out to obtain and verify the significant effect of Fin-Tech on the performance enhancement of commercial banks through coefficient regression. And Xu, Xiong, Jiang (2020) and others conducted the quantitative analysis of different provinces' Fin-Tech index from 2009 to 2017 at the macro level, using the markov chain, thayer index and moran index. The research mentioned above were based on two aspects, consisting of outer space discrepancy and internal dynamic development. Although the overall development level of Fin-Tech in our country was not high, but had risen steadily in recent 
years. Moreover, uneven level development was caused by regional differences and such situation needed to be improved according to circumstances.

Thirdly, the DEA model is boasted to analyze the development level of Fin-Tech in different regions of China. For the qualitative measurement of 12 provinces and autonomous regions in the west, $\mathrm{Wu}$ (2018) adopted the SE-DEA model and Malmquist model to select input indicators from the ways of public, market and overall Fin-Tech, as well as compared them with the eastern region to put forward suggestions for the development of Fin-Tech in the western region. On account of the Beijing-Tianjin-Hebei Yangtze River Delta, Pearl River Delta and other economic, Sun (2020) combined the static result of DEA-BCC model with the dynamic result of DEA-Malmquist model from the analysis of talent introduction, economic development, government support, scientific research atmosphere and so on many angles. Sun (2020) finally stated that Guangdong, Beijing and provinces which have more open economic policies or more powerful policy support remain at the optimum state of technical effectiveness. Different from other studies, PCA method was adopted to reduce the dimension of all input and output indicators by Zhou (2019)'s research on the GuangdongHong Kong-Macao Greater Bay Area, screening out the weak environmental impact and high degree of differentiation indexes. In result, poor efficiency of scale and redundancy in education funding were main factors leading to the low Fin-Tech efficiency in the Greater Bay Area.

To sum up, scholars have conducted relatively mature studies on the development and application of Fin-Tech, as well as the measurement of DEA efficiency model. However, there are few literatures about regional Fin-Tech by DEA efficiency model. Moreover, in terms of research methods, the threestage DEA model measurement model is mainly adopted while the combination of Malmquist index and DEA model is rarely used. When it comes to research subjects and levels, scholars mainly focus on the relatively macro level, such as the comparison between the eastern and western regions, the contrast between economic circles or provinces. There is still lack of studies focusing on more microscopic neighboring cities. As a consequence, this paper adopts the DEA-Malmquist index model to concentrate on the development of cities in the PRD from 2010 to 2018 and studies the reasons for different development level of Fin-Tech efficiency among cities, so as to perfect the researches in related fields.

\section{Empirical Research}

\subsection{Research Method}

Data Envelopment Analysis (DEA) was proposed by Charnes, Cooper and Rhodes(1978) and other professors of operations studies. It is an efficiency model for measuring multiple input and output indicators by establishing the corresponding production front based on the known data. Compared with other methods, the DEA model doesn't need to construct the functional relationship among various indicators, avoids calculating the weight among input indicators and reduces the steps of data dimensional conversion. More importantly, it can be combined with multiple output indicators for efficiency measurement. Traditional DEA models include CCR model and BCC model. CCR model assumes constant return to scale while BCC model assumes change of return to scale, containing FG, ST and other models proposed later. All models are established on the static condition, which means that analysis are conducted on the data from the same period and included in input orientation (under the condition of not changing output indicators, the decreasing degree of input indexes when it is effective) and output orientation (under the condition of not changing input indicators, the increasing degree of output indexes when it is effective).

Malmquist index was proposed by Malmquist (1953), and Caves et al. (1982) began to apply it to the measurement of productivity change. It was not until Rolffare (1994) that DEA was combined with the Malmquist index. The calculation of Malmquist index requires decision making units of two consecutive periods. Formula MT of Malmquist function is shown in Equation 1, where X and Y are input and output indexes respectively, and $\mathrm{D}$ is distance function: 
Volume 15 (2021)

$$
\begin{gathered}
M_{t}\left(x_{t+1}, y_{t+1}, x_{t}, y_{t}\right)=\frac{D_{t}\left(x_{t+1}, y_{t+1}\right)}{D_{t}\left(x_{t}, y_{t}\right)} \\
M_{t}\left(x_{t+1}, y_{t+1}, x_{t}, y_{t}\right)=\left[\frac{D_{t+1}\left(x_{t+1}, y_{t+1}\right)}{D_{t+1}\left(x_{t}, y_{t}\right)} \times \frac{D_{t}\left(x_{t+1}, y_{t+1}\right)}{D_{t}\left(x_{t}, y_{t}\right)}\right] \\
M t\left(x_{t+1}, y_{t+1}, x_{t}, y_{t}\right)=\text { effch * techch }=\text { pech *sech * techch }
\end{gathered}
$$

On this basis, Rolffare calculated the production efficiency ratio between $\mathrm{T}$ period and $\mathrm{T}+1$ period, assuming that returns to scale change. MT represents total factor productivity, EFFCH represents the change of technical efficiency, SECH represents the change rate of scale efficiency, PECH represents the variation of pure technical efficiency and Techch represents technological progress index.

This paper studies the efficiency of Fin-Tech in PRD from 2010 to 2018, using dynamic data of multiple periods and multi-dimensional input-output indicators. Therefore, DEAP software and output-oriented DEA-Malmquist model are adopted in this paper.

\subsection{Index Selection and Data Acquisition}

Summarizing the research of previous scholars, the author keeps to the following principles in the selection of input and output variables. First of all, the principle is objectivity. Then, all variables must be able to objectively and truly reflect the financial input and the output of scientific achievements with a significant impact Last but not least, multidimensionality is the key point. The Fin-Tech is described from many angles to make the result more accurate and stereoscopic. Therefore, this paper selects indicators as followed:

Table 1. Selected indicator

\begin{tabular}{|c|c|}
\hline Categories & Name \\
\hline Input variables & $\begin{array}{c}\text { enterprises above applicable size R\&D overall number of people/year } \\
\text { R\&D input /billion } \\
\text { Budgetary expenditures for regional science and technology / billion }\end{array}$ \\
\hline Output variables & $\begin{array}{c}\text { the number of granted patents/units } \\
\text { Output value of new industrial products above applicable size / billion }\end{array}$ \\
\hline
\end{tabular}

From the aspect of human input, total number of R\&D personnel in enterprises above designated size refers to all the personnel, including full-time and part-time personnel, who are involved in scientific and technological research each year. From the aspect of capital investment, R\&D investment refers to the research funds of enterprises, schools, scientific research institutes and other institutions. Its sources include government investment, enterprise investment, overseas investment and other funds, reflecting the degree of local economic openness and development to a certain extent. Regional science and technology budget expenditure reflects the extent of local government's support for local scientific research evolution and is the basic condition for promoting local science and technology progress.

The output variable is the number of local patent grants and the output value of new industrial products above designated size. Both are the direct embodiment of scientific achievements and innovation ability.

The above data are from 2010-2018 Statistical Yearbook of Guangdong Province, Statistical Bulletin of Science and Technology Investment of Guangdong Province, and local statistical yearbooks of nine cities in PRD. At the same time, considering that it takes a certain period for scientific and technological output to accumulate, and referring to the treatment of lag in previous 
literatures, the input data from 2010 to 2017 and output index from 2011 to 2018 are selected as the panel data processed by the DEA-Malmquist model.

\section{Result Analysis}

According to the calculation results of the DEAP model, the annual change of Fin-Tech efficiency in PRD from 2011 to 2018 are mainly obtained (Table 1) and the overall change of nine cities in PRD from 2011 to 2018 respectively (Table 2).

Table 2. Fin-Tech efficiency in PRD from 2011 to 2018

\begin{tabular}{|l|l|l|l|l|l|}
\hline year & effch & techch & pech & sech & tfpch \\
\hline 2012 & 0.928 & 1.136 & 0.984 & 0.943 & 1.054 \\
\hline 2013 & 1.081 & 1.184 & 1.021 & 1.058 & 1.279 \\
\hline 2014 & 0.997 & 1.136 & 0.999 & 0.999 & 1.343 \\
\hline 2015 & 0.976 & 0.804 & 0.992 & 0.984 & 0.785 \\
\hline 2016 & 0.758 & 1.320 & 0.830 & 0.914 & 1.001 \\
\hline 2017 & 1.117 & 0.679 & 1.143 & 0.978 & 0.759 \\
\hline 2018 & 0.911 & 0.832 & 0.934 & 0.975 & 0.757 \\
\hline mean & 0.096 & 1.012 & 0.982 & 0.978 & 0.972 \\
\hline
\end{tabular}

Table 3. Fin-Tech efficiency of cities in PRD from 2011 to 2018

\begin{tabular}{|c|c|c|c|c|c|}
\hline city & effch & techch & pech & sech & tfpch \\
\hline Shenzhen & 1.000 & 1.010 & 1.000 & 1.000 & 1.010 \\
\hline Guangzhou & 0.954 & 1.026 & 1.000 & 0.954 & 0.978 \\
\hline Foshan & 0.936 & 1.016 & 0.958 & 0.977 & 0.950 \\
\hline Zhongshan & 0.962 & 0.993 & 0.977 & 0.984 & 0.955 \\
\hline Huizhou & 0.928 & 1.000 & 0.966 & 0.961 & 0.929 \\
\hline Dongguan & 0.941 & 1.018 & 0.963 & 0.977 & 0.958 \\
\hline Zhuhai & 1.000 & 1.035 & 1.000 & 1.000 & 1.035 \\
\hline Jiangmen & 0.957 & 0.999 & 0.981 & 0.976 & 0.956 \\
\hline Zhaoqing & 0.969 & 1.010 & 0.996 & 0.973 & 0.978 \\
\hline mean & 0.960 & 1.012 & 0.982 & 0.978 & 0.972 \\
\hline
\end{tabular}

As shown in Table 1, the average value of total factor productivity (TFPCH) in the PRD is slightly lower than 1, indicating that TFPCH presents a slight downward trend. In terms of comprehensive technical progress index (Techch), pure technical efficiency index (PECH) and scale efficiency index $(\mathrm{SECH})$, only the average value of Techch is slightly greater than 1 . As for specific years, the efficiency is relatively effective in 2011-2014 and 2015-2016. During the period of 2014-2015 and 2016-2018, they were in a relatively ineffective state, reflecting the general trend of "upwarddownward-upward-down".

The main factor affecting total factor productivity (TFP) is the technical progress index (Techch). When the Techch is relatively effective, the total efficiency is relatively effective. From the perspective of the author, the main factors are as followed. First of all, it is the policy regulations. In 2011, the government promulgated the "12th Five-Year Plan" and issued the "12th Five-Year Special Plan for the Development of Science and Technology in Modern Service Industry" as well, which emphasized a new model about how to expand financial industry with promoting the development of the technical industry and the transformation of achievements. In 2016, the "13th Five-Year Plan" was issued. In the same year, the "13th Five-Year Plan for National Science and Technology Innovation" was released, which clearly promoted the combination of technology and finance through the innovation of Fin-Tech products and services. Secondly, economic development is the principal influence factor. Since the trough of the economic cycle ended in 2010 and the world economic crisis in 2008, China's economy began to pick up. The absolute increment after 2010 is higher than that before 2010 for both national GDP and Guangdong GDP. With the support of both policy and economy, local governments strengthen the development and combination of finance and technology 
from various aspects, such as building technical parks, introducing high-tech enterprises and highend talents, increasing the budget for scientific research and facilitating loans for small and mediumsized enterprises. Taking the total R\&D expenditure as an example, from 2010 to 2018, the average increase in the PRD was about $300 \%$ with the $172 \%$ average increase in Zhongshan and $489 \%$ growth rate in Huizhou. When the technical progress index is relatively ineffective, the total efficiency of Fin-Tech is in a negative growth state. In the data collected in this paper, the economic capital investment still maintains a high growth rate, but the human capital investment growth rate is slow with negative growth increase. As one of the output indicators, the growth rate of new product's output value in industrial enterprises above applicable size is much lower than the growth rate of the investment of economic capital. The possible reason is that after 2015, the world economic situation is not optimistic, with the economic growth rate of various countries declining and the inflation rate falling continuously, which means that the global supply exceeds the demand and the international trade has negative increase. Therefore, although China's GDP is maintaining growth, most enterprises are facing operational difficulties and talents cannot play an effective role. In addition, high-tech enterprises bore the brunt of China's trade war with the United States in 2018. In Shenzhen, one of the cities heavily populated with high-tech companies, the output value of new products grew at $3.14 \%$ in 2017-2018, much slower than the average growth rate of $17.6 \%$ in $2011-2018$.

As can be seen from Table 2, among nine cities in the PRD, only Shenzhen and Zhuhai are FinTech effective from 2010 to 2018 . Combined with the DEAP calculation results, the specific situation of nine cities is the same as the overall change trend, which shows that technological progress index is still the most influential factor and the trend of TFP is in line with the fluctuation of "upwarddownward-upward-down".

The author has mentioned the reasons for the trend of TFP change above. Integrated with the specific situation of the PRD, the author's analysis of Shenzhen and Zhuhai's Fin-Tech efficiency are in effective status are as followed.

In order to promote the rapid development of the PRD, the Guangdong Provincial Government has successively proclaimed the Outline of the Reform and Development Plan for the Pearl River Delta Region (2008-2020) and the Action Plan for Promoting the Integration of Science, Technology and Innovation in the Pearl River Delta Region (2014-2020). The State Council also issued the Implementation Plan for the Construction of Pearl River Delta National Independent Innovation Demonstration Zone (2016-2020) to build a "1+1+7" system with Guangzhou and Shenzhen as the core.

Based on the analysis of the collected data and model results, Guangzhou and Shenzhen have become the core of the PRD, where the input index and output index are far higher than the other seven cities. However, in terms of Fin-Tech efficiency, Shenzhen's development speed exceeds Guangzhou's. From the beginning, Shenzhen's technical efficiency is not only lower than Guangzhou's, but also in a state of negative growth. In 2018, despite the severe impact of the external environment, Shenzhen's technical efficiency remained stable and did not decline. This may be related with two factors. One is the introduction of talents. Taking the third quarter of 2019 as an example, about $48 \%$ of talents in the Greater Bay Area went to Shenzhen and $29 \%$ to Guangzhou. The other is industrial distribution. In addition to the intensive distribution of high-tech enterprises, financial enterprises are also one of the pillar industries in Shenzhen. The pillar industries of Guangzhou are only information, electronics, automobile and other industrial enterprises. Compared with other cities, Zhuhai can achieve effective state, mainly because it can achieve the same output increase ratio under the condition that the growth rate of human input index is not large, when other cities only rely on the growth of capital input. Zhuhai is more suitable for the local pillar industries in terms of talent introduction, so that the technical efficiency can be guaranteed. 


\section{Policy Suggestion}

Based on the data of PRD from 2010 to 2018, combined with the calculation and analysis of DEAMalmquist model, it can be concluded that the overall efficiency of Fin-Tech in the PRD is in a state of continuous decline, mainly affected by the technical efficiency index. Only Shenzhen and Zhuhai can maintain relative efficiency. In view of the above conclusions, this paper puts forward some suggestions, hoping to promote the better development of Fin-Tech in the PRD:

Firstly, it is necessary to break down administrative barriers between cities and promote coordinated development within the PRD. As mentioned above, on account of the statistical yearbooks and model results of various cities, the PRD has formed a "1+1+7" system with Guangzhou and Shenzhen as the core. In terms of overall development, it is far ahead of other cities in culture, education, technology, finance, industry, tourism and other aspects. However, among Jiangmen, Zhuhai and Zhaoqing, which are relatively backward in development, the Fin-Tech efficiency in Zhuhai can be in a relatively effective state. Therefore, local governments should break the barriers between cities and promote all-round exchanges and cooperation. They will establish a public service platform, share high-quality local resources, and enrich the content and methods of services. What's more, the government should effectively play the role of the system with Guangzhou and Shenzhen as the core, radiate and drive the development of other cities. Then, it strengthens the support for relatively backward cities such as Jiangmen and Zhaoqing, jointly builds the relevant systems of FinTech and studies the development strategies of Shenzhen and Zhuhai. Local governments should take measures according to local conditions and improve local policies.

Secondly, improving financial policies and accelerate the integration of financial and technology enterprises are helpful. Small and medium-sized enterprises remain the mainstay of technology enterprises in the PRD. However, small and medium-sized enterprises are likely to face difficulties brought by capital in the stages of exploring more mature research and development technologies, accelerating the transformation of scientific research achievements and perfecting efficient operation systems. As an important economic center of the PRD, the government should make rational use of it. In terms of fiscal policies, the government should increase investment in scientific research funds and reduce or exempt taxes. At the same time, relying on the relatively perfect capital market, the government should give corresponding policy guidance, so that the traditional financing institutions, such as banks, and the current social investment institutions, such as angel investment, venture capital and other enterprises obtain corresponding support and convenience. While promoting the development of enterprises, it also disperses the relevant risks.

Thirdly, the government has the obligation to strengthen investment in human resources and cultivate innovative talent teams. The internal driving force of the development of science and technology enterprises needs the appropriate high-level talents. Combined with the collected data and measurement results, the technical efficiency index is an important element affecting the relative inefficiency of total factor productivity. After 2015, the growth rate of human resource investment is significantly lower than that of capital investment. In order to strengthen human resources investment, on the one hand, it is imperative to improve the talent introduction system to attract high-level talents to settle in the PRD. On the other hand, the government should strengthen investment in education, promote the evolution of universities, train local talents, strengthen the bond of industry and learning, and build the research-friendly atmosphere.

\section{References}

[1] Sfinga, I., \& Niklis, D. (2011). EVALUATION OF BANK EFFICIENCY WITH THE DEA APPROACH: A COMPARISON OF EUROPEAN COMMERCIAL BANKS. Journal of Computational Optimization in Economics and Finance, 3(2), 115-131. Retrieved from https://search.proquest.com/scholarly-journals/ evaluation- bank-efficiency-with-dea-approach/docview/1728422463/se-2?accountid=162699. 
[2] Branda, M., \& Kopa, M. (2012). DEA-risk efficiency and stochastic dominance efficiency of stock indices*. Finance a Uver, 62(2), 106-124. Retrieved from https://search.proquest.com/scholarly-journals/ dea-risk-efficiency-stochastic-dominance-stock/docview/1020691776/se-2?accountid=162699.

[3] Seol, H., Lee, H., Kim, S., \& Park, Y. (2008). The impact of information technology on organizational efficiency in public services: A DEA-based DT approach. The Journal of the Operational Research Society, 59(2), 231-238. Doi: http://dx.doi.org/10.1057/palgrave.jors.2602453.

[4] Jiang Ruixue. (2020). Research on enterprise diversification, resource distribution and wealth distribution based on DEA (Ph.D. thesis, University of Science and Technology of China).

[5] Ren Yuquan. Research on the financing efficiency of listed companies in the new energy automobile industry chain based on the DEA model [D]. Zhejiang University, 2020.

[6] Liu Zhaohong. (2020). Research on the mitigation effect of the development of science and technology finance on the financing constraints of SMEs.

[7] Wang Juan. (2020). Research on the impact of financial technology on the performance of commercial banks.

[8] Xu Yuming, Xiong Qizhe and Jiang Yun (2020). Analysis of the measurement and related characteristics of the science and technology financial development index. Finance and Economics (12), 42-48. doi: 10.19622 / j.cnki.cn36-1005 / f.2020.12.005.

[9] $\mathrm{Wu}$ Xuefen. Research on the efficiency of science and technology finance in the western region [D]. Shenzhen University, 2018.

[10] Sun Zhongyan. (2020). An empirical study on the efficiency and influencing factors of domestic regional science and technology finance.

[11] Zhou Yuan. Research on the efficiency of science and technology finance in the Guangdong-Hong KongMacao Greater Bay Area [D]. Shenzhen University, 2019.

[12] Yan Bo, Du Jun \& Pan Hong. (2019). The status quo, problems and countermeasures of scientific and technological collaborative innovation in the Pearl River Delta region. Science and Technology Management Research (01), 87-96. Doi.

[13] Huang Ruifen \& Qiu Mengyuan. (2016). Internal scientific financial efficiency evaluation based on Malmquist index and SFA model. Science and Technology Management Research (20), 43-48. doi: CNKI: SUN: KJGL.0.2016-20-009.

[14] Deng Xue, Chen Chuangjie, Shen Lu \& Liang Ying. (2020). Performance evaluation of technology finance based on Malmquist-DEA model-take Guangdong Province as an example. Science and Technology Management Research (21), 64-72. doi: CNKI: SUN: KJGL.0.2020-21-008.

[15] Zhang Peng, Li Linxin and Zeng Yongquan. (2021). Research on the evaluation of scientific and technological innovation efficiency in the Guangdong-Hong Kong-Macao Greater Bay Area based on DEA-Malmquist index. Industrial Technology Economy (02), 12-17. doi: CNKI: SUN: GHZJ.0.2021-02002. 